\title{
A Retrospective Review of Unicompartmental Knee Arthroplasty Functional Outcome and the Incidence of Medial Meniscus Posterior Root Tear in Spontaneous Osteonecrosis of the Knee
}

\section{PO-JU WU}

Mackay Memorial Hospital https://orcid.org/0000-0003-0596-0978

TsungYu Lin

Mackay Memorial Hospital

Yung Chang Lu ( $\sim$ james01685@gmail.com )

Mackay Memorial Hospital

\section{Research Article}

Keywords: Unicompartmental knee arthroplasty, Spontaneous osteonecrosis, Knee osteonecrosis, Medial meniscus posterior root tear

Posted Date: November 10th, 2020

DOI: https://doi.org/10.21203/rs.3.rs-70036/v3

License: (c) (1) This work is licensed under a Creative Commons Attribution 4.0 International License.

Read Full License 


\section{Abstract}

\section{Background:}

Spontaneous osteonecrosis of the knee (SONK) can lead to severe knee osteoarthritis predominantly localized to the medial compartment. We aimed to determine whether unicompartmental knee arthroplasty was an effective treatment for primary SONK.

Methods: We analyzed the functional outcomes in 23 patients with SONK (with a magnetic resonance imaging [MRI]-confirmed diagnosis) who underwent UKA at a single center. The mean follow-up time was 67 months post-UKA.

Results: Significant improvements in function were indicated by reduced Oxford Knee and Visual Analogue Scale scores after UKA, and there were no specific complications after the procedures. The incidence of MRI-identified medial meniscus posterior root tear (MMPRT) was 69.6\% (16/23).

Conclusion: Unicompartmental arthroplasty for SONK is less destructive to the native knee structure than total knee arthroplasty, but can achieve comparable prognosis with strict patient selection. While the precise etiology of SONK is unknown, one theory posits that a MMPRT may change the biomechanical circumstances of the knee joint, leading to osteonecrosis. Although not confirmatory, the high prevalence rate suggests that MMPRT may have a key role in the development of SONK. UKA is an effective treatment option for SONK, resulting in significant functional improvement. Long-term (>10 years) outcomes should be investigated.

\section{Background}

Osteonecrosis of the knee is caused by diverse etiologies [1]. The condition can be divided into three subtypes: spontaneous, secondary, and post-arthroscopic [2]. Spontaneous osteonecrosis of the knee (SONK) may be induced by microfractures of the subchondral bone whereby it can lead to joint space narrowing or end-stage knee arthritis [3]. SONK is the most common form of osteonecrosis, with the highest prevalence in women and people aged over 60 years. The condition is usually unilateral and predominantly localized to the medial compartment [4].

Treatment options for SONK are based on specific symptoms and the disease stage. Non-surgical treatments for pre-collapse SONK include protected weight bearing and analgesic usage as needed [5]. Surgical treatments included joint-preserving procedures and arthroplasty.

Unicompartmental knee arthroplasty (UKA) is considered as a less destructive treatment option for SONK as it preserves more the native knee and proprioception compared to total knee arthroplasty (TKA) [6]. When osteoarthritis is involved in more than one compartment, TKA is the preferred option.

Advances in both prosthesis design and surgical techniques have led to UKA becoming an increasingly valid surgical option for SONK. However, because of the small sample sizes used in previous studies, it 
remains controversial whether UKA is comparable to TKA for the treatment of SONK $[7,8,9,10]$.

Therefore, in this study, we analyzed clinical outcomes of UKA for primary SONK at a mean follow-up period of 67 months. Because it has been proposed that medial meniscus posterior root tear (MMPRT) may play an important part in the development of SONK, we also investigated the incidence of MMPRT in this cohort of SONK patients.

\section{Methods}

We conducted a retrospective chart review of patients who underwent UKA at the Department of Orthopedics, MacKay Hospital (Taipei, Taiwan). We reviewed the charts of all patients $(n=81)$ who underwent UKA using a Physica ZUK (Lima Corporate, Udine, Italy) at our hospital between 2013 and 2014 and followed up for at least 5 years after surgery. The inclusion criterion was patients with primary SONK at the medial femoral condyle of the knee. The exclusion criteria were as follows: 1 . singlecompartment osteoarthritis without osteonecrosis lesion; 2 . secondary osteonecrosis; 3 . infection; 4. inflammatory arthritis; 5 . flexion contracture; 6 . ligament instability; and 7. SONK combined with multiple compartment osteoarthritis (Fig. 1). Surgeries were performed in a standard manner with cemented components for all cases. During the procedure, the necrotic bone lesion was removed as possible, with the residual healthy bone serving as the base for cement construction (Fig. 2).

During routine care, clinical and radiographic data were collected before and after the operation, and follow-ups were scheduled at 1,3 , and 12 months, respectively, and subsequently once every year. Medical records were reviewed thoroughly to confirm revision, re-operation, and complication rates. The Oxford Knee Score (OKS) [11] and Visual Analogue Scale (VAS) for pain were used to analyze postoperative clinical outcomes. Clinical data for all patients are shown in Table 1. In total, six men and 17 women were included in the study, with an age range of 54-80 years (mean 68.9 years) and body mass index (BMI) range of $21.7-33.2 \mathrm{~kg} / \mathrm{m}^{2}$ (mean $26.5 \mathrm{~kg} / \mathrm{m}^{2}$ ).

Radiographic images were used to diagnose disease stage, ranging from early stage pre-collapse to late stage subchondral collapse. The Ficat stage was classified using plain x-rays in the A-P view (Table 2).

Pre-operative magnetic resonance imaging (MRI) was used to detect meniscal lesions as well as the size and area of necrosis. Necrosis percentage was measured using the condylar ratio in the AP direction, and the volume of SONK was calculated by multiplying the greatest AP width, lateral width, and lesion height in the sagittal view (Table 2).

Based on a previous report [12], MMPRT was defined by the presence of both following MRI findings:

(1) Interruption of the medial meniscus at the posterior horn in the coronal or axial plane, known as the "cleft sign" (Fig. 3a) and 
(2) An empty image of the medial meniscus at the posterior horn in the sagittal plane, known as the 'ghost sign (Fig. 3b).

All statistical data were analyzed using the SPSS version 22.0 software ${ }^{\circledR}$ (IBM Corporation, Armonk, NY, USA). The pre- and postoperative OKS and VAS were compared using paired T-tests. P-values $<0.05$ were considered significant.

This study was conducted in accordance with the Declaration of Helsinki and the protocol was approved by the Ethics Committee of the MacKay Hospital (Project identification code: 19MMHIS161e). All patients provided informed consent for inclusion in the study.

\section{Results}

Twenty-three UKA knees due to SONK were included in the final analysis. The mean follow-up period was 67 months (range: 60 to 76 months). There were no severe complications, such as deep vein thrombosis, prosthetic infection, fat embolism, or death in our cohort. MRI findings for all patients are listed in Table 2. The mean condylar ratio was $62.2 \%$ (range: $44.0-87.9 \%$ ) in the AP direction. MRI-confirmed MMPRT was identified in $69.5 \%(16 / 23)$ of all SONK cases.

The mean clinical outcome scores for pre-op and post-op VAS were 8.04 (range: 7.0-9.0) and 2.61 (range: 1.0-4.0), respectively. The mean clinical outcome score for the pre- and post-operative OKS was 40.65 (range: $32.0-48.0$ ) and 16.73 (range: 12.0-32.0), respectively (Table 3). Comparing clinical outcomes post- versus pre-surgery, the post-operative VAS and OKS were significantly lower than the preoperative scores (both $p<0.001)$ (Table 4).

\section{Discussion}

Uni-compartment knee arthroplasty is an effective treatment for SONK. In this study, knee pain was alleviated and the function of the knee improved after UKA. SONK is a common condition that can be treated non-operatively or with joint-preserving surgical intervention [13, 14]. SONK can progress to articular collapse and end-stage joint osteoarthritis despite medical intervention. Knee arthroplasty for SONK includes both TKA and UKA approaches; however, it remains controversial which approach results in the best surgical outcome for SONK. The indications for UKA are much stricter than those of TKA for patients with osteonecrosis. UKA is utilized in single-compartment osteonecrosis without degenerative changes in other compartments. Intact ligament structures with virtually normal alignment are also required. Several previous studies reported that TKA gave better outcomes than UKA $[14,15]$. However, using current surgical techniques, UKA can preserve the anterior cruciate ligament and non-involved cartilage in other knee compartments, all of which are sacrificed in TKA. As a result, UKA is considered a better option for SONK patients with only single compartment involvement. In previous studies, UKA had a higher risk of revision and worse clinical outcomes than TKA for single compartment osteoarthritis [16, 17]. Using a modern implant design and modified surgical techniques, Myers et al. found that inadequate 
patient selection and improper surgical indication wound resulted in high revision rates and poor clinical outcomes $[7,18,19]$. Kozinn et al. concluded that UKA was contraindicated in patients with inflammatory arthritis, tricompartmental knee arthritis, fixed varus deformity greater than $10^{\circ}$, fixed valgus deformity greater than $5^{\circ}$, flexion contracture greater than $15^{\circ}$, and knee instability without an intact anterior cruciate ligament [20].

A prospective study of SONK treated with UKA showed significant improvement in OKS post-operatively. UKA appears to be the treatment of choice with the advantage of minimizing bone stock destruction, decreasing the use of bone cement, and better preservation of knee function in comparison to outcomes following TKA [21].

Chalmers et al. found that the success rates of UKA without revision surgery at 5 and 10 years postoperatively were $89 \%$ and $76 \%$, respectively. Three cases $(7.1 \%)$ were converted to TKA, one for development of lateral osteonecrosis (secondary osteonecrosis due to steroid treatment) and two for lateral compartment degeneration [22]. Secondary osteonecrosis of the knee can be caused by alcohol abuse, high-dose systemic corticosteroid use, or other direct cause [2]. Misdiagnosed secondary osteonecrosis was a risk factor for UKA success and further revision surgery.

Polyethylene wear is another issue, and some studies reported that fixed-bearing UKA may produce substantial wear due to malposition. The incidence of polyethylene wear is rare in mobile-bearing UKA because this prosthesis preserves more of the natural kinematics of the knee. However, all of our cases were conducted with fixed-bearing prostheses, and none showed obvious signs of wear on follow-up radiographic images [23].

Because the precise etiology of SONK is currently unknown, effective management of the condition remains challenging for orthopedists. In the past, SONK was thought to result from focal ischemia that caused bone necrosis. Another hypothesis was that SONK may be caused by subchondral insufficiency fractures. Subchondral insufficiency fractures may produce fluid accumulation in the bone marrow, bringing on focal ischemia and ultimately necrosis [3,24]. Subchondral insufficiency fractures may be attributed to pathological lesions, including osteoporosis, meniscus tear, or meniscectomy.

A recent study in support of this alternative hypothesis demonstrated that meniscus tears are related to cartilage destruction in the knee joint. Allaire et al. proposed that MMPRT may alter the knee's natural biomechanics and increase the peak contact pressure in the joint. The authors even raised the possibility that the effect after MMPRT injury was comparable to the patients' status-post total medial meniscectomy [25]. The incidence of MRI-identified MMPRT was $69.6 \%$ (16/23 cases) in our study cohort. A retrospective analysis by Robertson et al. found a similar high incidence of MRI-identified MMPRT ( $80 \%$ of patients) in SONK. They proposed that femoral overload with increasing interosseous pressure by meniscal discontinuity is one of the causes of SONK [26]. In comparison to patients with knee osteoarthritis, the incidence of MMPRT and the level of posterior tibia slope were higher in SONK patients. Yamagami et al. proposed that MMPRT and higher posterior tibia slope have a greater 
association with SONK development [12]. Although the definite mechanism remains unknown, many studies have proposed that MMPRT has a key role in the development of SONK.

This study has limitations. First, we utilized a retrospective design with a small number of patients. Second, only short-term follow-up was available. No long-term radiographic outcomes or complication rates from polyethylene wear or implant loosening were available for assessment. Future long-term prospective studies are needed to fully understand the functional outcomes and complication rates.

\section{Conclusions}

UKA is an effective treatment option for SONK, resulting in significant functional improvement. Long-term outcomes need to be investigated. A high incidence of MMPRT was noted in our patient cohort, suggesting that the relationship between SONK and MMPRT requires further research.

\section{Abbreviations}

Spontaneous osteonecrosis of the knee (SONK)

Unicompartmental knee arthroplasty (UKA)

Total knee arthroplasty (TKA)

Medial meniscus posterior root tear (MMPRT)

Visual Analogue Scale (VAS)

Oxford Knee Score (OKS)

Magnetic resonance imaging (MRI)

\section{Declarations}

\section{Ethics approval and consent to participate}

This study was conducted in accordance with the 1964 Declaration of Helsinki and the protocol was approved by the Ethics Committee of the Mackay Memorial Hospital (Project identification code: 19MMHIS161e).

\section{Consent for publication}

All patients provided informed consent for inclusion in the study.

\section{Availability of data and material}

The authors confirm that the data supporting the findings of this study are available within the article. 


\section{Competing interests}

The authors declare that they have no competing interests.

\section{Funding}

The author(s) received no financial support for the research, authorship, and/or publication of this article.

\section{Authors' contributions}

Po-Ju Wu and Yung Chang Lu designed the study. Po-Ju Wu performed the postoperative follow-up for the patients and collected the clinical data. Tsung Yu Lin analysed the statistics data. Po-Ju Wu prepared the manuscript. All authors read and approved the final manuscript.

\section{Acknowledgements}

I would like to thank all my colleagues in the orthopaedic department of Mackay Memorial Hospital who contributed to this study. I want to give my special thanks to Dr. Yung Chang Lu for his collaboration during preliminary investigations and data collection. I am also grateful to Dr. Tsung Yu Lin for his help with the statistics in the research and the constructive review of the earlier version of this article.

\section{References}

1. Shah KN, Racine J, Jones LC, Aaron RK. Pathophysiology and risk factors for osteonecrosis. Curr Rev Musculoskelet Med. 2015;8(3):201-9.

2. Mont MA, Baumgarten KM, Rifal A, Bluemke DA, Jones LC, Hungerford DSJJ. Atraumatic osteonecrosis of the knee. 2000;82(9):1279.

3. Yamamoto T, Bullough PG. Spontaneous osteonecrosis of the knee: the result of subchondral insufficiency fracture. The Journal of bone and joint surgery American volume. 2000;82(6):858-66.

4. Mears SC, McCarthy EF, Jones LC, Hungerford DS, Mont MA. Characterization and pathological characteristics of spontaneous osteonecrosis of the knee. lowa Orthop J. 2009;29:38-42.

5. Yates PJ, Calder JD, Stranks GJ, Conn KS, Peppercorn D, Thomas NP. Early MRI diagnosis and nonsurgical management of spontaneous osteonecrosis of the knee. Knee. 2007;14(2):112-6.

6. Heyse TJ, Khefacha A, Fuchs-Winkelmann S, Cartier P. UKA after spontaneous osteonecrosis of the knee: a retrospective analysis. Arch Orthop Trauma Surg. 2011;131(5):613-7.

7. Myers TG, Cui Q, Kuskowski M, Mihalko WM, Saleh KJ. Outcomes of total and unicompartmental knee arthroplasty for secondary and spontaneous osteonecrosis of the knee. The Journal of bone and joint surgery American volume. 2006;88 Suppl 3:76-82.

8. Ye J-X, Yang X-F, Lian-Sheng D, Feng-Lai Y, Rui-Sheng X, Yang Z-G. Compared to TKA or HTO, will you choose UKA to treat medial compartmental knee osteoarthritis? A review paper on UKA. J Phys. 2018;2(1):26. 
9. Choy WS, Kim KJ, Lee SK, Yang DS, Kim CM, Park JS. Medial unicompartmental knee arthroplasty in patients with spontaneous osteonecrosis of the knee. Clin Orthop Surg. 2011;3(4):279-84.

10. Zhang Q, Guo W, Liu Z, Cheng L, Yue D, Zhang N. Minimally invasive unicompartmental knee arthroplasty in treatment of osteonecrosis versus osteoarthritis: a matched-pair comparison. Acta Orthop Belg. 2015;81(2):333-9.

11. Murray D, Fitzpatrick R, Rogers K, Pandit H, Beard D, Carr A, et al. The use of the Oxford hip and knee scores. 2007;89(8):1010-4.

12. Yamagami R, Taketomi S, Inui H, Tahara K, Tanaka S. The role of medial meniscus posterior root tear and proximal tibial morphology in the development of spontaneous osteonecrosis and osteoarthritis of the knee. Knee. 2017;24(2):390-5.

13. Mont MA, Marker DR, Zywiel MG, Carrino JA. Osteonecrosis of the knee and related conditions. The Journal of the American Academy of Orthopaedic Surgeons. 2011;19(8):482-94.

14. Marmor L. Unicompartmental arthroplasty for osteonecrosis of the knee joint. Clinical orthopaedics and related research. 1993(294):247-53.

15. Radke S, Wollmerstedt N, Bischoff A, Eulert J. Knee arthroplasty for spontaneous osteonecrosis of the knee: unicompartimental vs bicompartimental knee arthroplasty. Knee Surg Sports Traumatol Arthrosc. 2005;13(3):158-62.

16. Baker PN, Petheram T, Avery PJ, Gregg PJ, Deehan DJ. Revision for unexplained pain following unicompartmental and total knee replacement. The Journal of bone and joint surgery American volume. 2012;94(17):e126.

17. Niinimaki T, Eskelinen A, Makela K, Ohtonen P, Puhto AP, Remes V. Unicompartmental knee arthroplasty survivorship is lower than TKA survivorship: a 27-year Finnish registry study. Clinical orthopaedics and related research. 2014;472(5):1496-501.

18. Steele RG, Hutabarat S, Evans RL, Ackroyd CE, Newman JH. Survivorship of the St Georg Sled medial unicompartmental knee replacement beyond ten years. The Journal of bone and joint surgery British volume. 2006;88(9):1164-8.

19. Barrett WP, Scott RD. Revision of failed unicondylar unicompartmental knee arthroplasty. The Journal of bone and joint surgery American volume. 1987;69(9):1328-35.

20. Kozinn SC, Scott RD. Surgical treatment of unicompartmental degenerative arthritis of the knee. Rheumatic diseases clinics of North America. 1988;14(3):545-64.

21. Ma T, Tu Y, Xue H, Wen T, Mei J. Unicompartmental knee arthroplasty for spontaneous osteonecrosis. J Orthop Surg (Hong Kong). 2017;25(1):2309499017690328.

22. Chalmers BP, Mehrotra KG, Sierra RJ, Pagnano MW, Taunton MJ, Abdel MP. Reliable outcomes and survivorship of unicompartmental knee arthroplasty for isolated compartment osteonecrosis. The bone \& joint journal. 2018;100-b(4):450-4.

23. Kwon OR, Kang KT, Son J, Kwon SK, Jo SB, Suh DS, et al. Biomechanical comparison of fixed- and mobile-bearing for unicomparmental knee arthroplasty using finite element analysis. J Orthop Res. 2014;32(2):338-45. 
24. Akamatsu Y, Mitsugi N, Hayashi T, Kobayashi H, Saito T. Low bone mineral density is associated with the onset of spontaneous osteonecrosis of the knee. Acta Orthop. 2012;83(3):249-55.

25. Allaire R, Muriuki M, Gilbertson L, Harner CD. Biomechanical consequences of a tear of the posterior root of the medial meniscus. Similar to total meniscectomy. The Journal of bone and joint surgery American volume. 2008;90(9):1922-31.

26. Robertson DD, Armfield DR, Towers JD, Irrgang JJ, Maloney WJ, Harner CD. Meniscal root injury and spontaneous osteonecrosis of the knee: an observation. The Journal of bone and joint surgery British volume. 2009;91(2):190-5.

\section{Tables}

Table 1 Clinical data of all patients 


\begin{tabular}{|c|c|c|c|c|}
\hline Case no. & Sex & $\begin{array}{c}\text { Age } \\
\text { (years) }\end{array}$ & $\begin{array}{c}\text { BMI } \\
\left(\mathrm{kg} / \mathrm{m}^{2}\right)\end{array}$ & $\begin{array}{l}\text { Follow-up } \\
\text { (months) }\end{array}$ \\
\hline 1 & F & 71 & 30.1 & 76 \\
\hline 2 & M & 68 & 26.6 & 74 \\
\hline 3 & $\mathrm{~F}$ & 62 & 28.5 & 74 \\
\hline 4 & F & 60 & 26.7 & 72 \\
\hline 5 & F & 75 & 28.1 & 71 \\
\hline 6 & F & 80 & 26.3 & 71 \\
\hline 7 & F & 77 & 24.9 & 70 \\
\hline 8 & M & 60 & 27.7 & 69 \\
\hline 9 & M & 72 & 22.8 & 69 \\
\hline 10 & F & 68 & 24.3 & 69 \\
\hline 11 & F & 78 & 25.1 & 68 \\
\hline 12 & M & 64 & 23.7 & 67 \\
\hline 13 & F & 69 & 21.7 & 67 \\
\hline 14 & F & 73 & 23.9 & 66 \\
\hline 15 & F & 54 & 22.3 & 65 \\
\hline 16 & F & 67 & 23.3 & 65 \\
\hline 17 & F & 69 & 36 & 64 \\
\hline 18 & F & 64 & 30.5 & 64 \\
\hline 19 & F & 71 & 25.4 & 62 \\
\hline 20 & M & 70 & 28 & 62 \\
\hline 21 & F & 77 & 24.4 & 61 \\
\hline 22 & M & 77 & 25.2 & 60 \\
\hline 23 & F & 59 & 33.2 & 60 \\
\hline
\end{tabular}

Table 2 Radiographic data 


\begin{tabular}{|c|c|c|c|c|}
\hline Case No. & Ficat stage & Condylar ratio (\%) & SONK volume $\left(\mathrm{cm}^{3}\right)$ & Meniscus tear in MRI \\
\hline 1 & II & 61.4 & 5.833 & $\operatorname{MMPRT}(+)$ \\
\hline 2 & II & 67.4 & 4.118 & $\operatorname{MMPRT}(+)$ \\
\hline 3 & II & 44.0 & 1.349 & $\operatorname{MMPRT}(+)$ \\
\hline 4 & III & 62.7 & 4.147 & $\operatorname{MMPRT}(+)$ \\
\hline 5 & III & 87.9 & 6.881 & $\operatorname{MMPRT}(+)$ \\
\hline 6 & II & 63.1 & 5.017 & $\operatorname{MMPRT}(+)$ \\
\hline 7 & III & 67.2 & 4.672 & $\operatorname{MMPRT}(+)$ \\
\hline 8 & III & 53.2 & 7.977 & $(-)$ \\
\hline 9 & I & 54.8 & 2.786 & $(-)$ \\
\hline 10 & I & 65.4 & 14.114 & MMPRT(+) \\
\hline 11 & III & 68.3 & 12.41 & $\operatorname{MMPRT}(+)$ \\
\hline \multirow[t]{2}{*}{12} & II & 48.0 & 1.854 & Medial meniscus anterior horn tear, \\
\hline & & & & MMPRT(-) \\
\hline 13 & II & 61.5 & 5.707 & $\operatorname{MMPRT}(+)$ \\
\hline 14 & I & 56.6 & 10.731 & Medial meniscus tear \\
\hline 15 & I & 62.4 & 9.309 & $\operatorname{MMPRT}(+)$ \\
\hline 16 & III & 59.5 & 10.731 & $\operatorname{MMPRT}(+)$ \\
\hline 17 & III & 65.6 & 4.146 & $\operatorname{MMPRT}(+)$ \\
\hline 18 & II & 71.2 & 20.694 & $\operatorname{MMPRT}(+)$ \\
\hline \multirow[t]{2}{*}{19} & III & 63.9 & 11.727 & Medial meniscus anterior horn tear, \\
\hline & & & & MMPRT(-) \\
\hline \multirow[t]{2}{*}{20} & II & 52.5 & 2.829 & Medial meniscus anterior horn tear, \\
\hline & & & & MMPRT(-) \\
\hline 21 & III & 66.7 & 9.108 & $\operatorname{MMPRT}(+)$ \\
\hline 22 & II & 48.8 & 7.749 & $(-)$ \\
\hline \multirow[t]{2}{*}{23} & II & 78.9 & 18.022 & $\operatorname{MMPRT}(+)$ \\
\hline & & Mean:62.2\% & & $\operatorname{MMPRT}(+): 16 / 23$ cases \\
\hline
\end{tabular}

SONK: spontaneous osteonecrosis of the knee; MMPRT: medial meniscus posterior root tear

Table 3 SONK patient outcomes 


\begin{tabular}{llll}
\hline & Minimum & Maximum & Mean \pm std. \\
\hline Pre-op VAS & 7.0 & 9.0 & $8.04 \pm 0.77$ \\
Post-op VAS & 1.0 & 4.0 & $2.61 \pm 0.72$ \\
Pre-op OKS & 32 & 48 & $40.65 \pm 4.28$ \\
Post-op OKS & 12 & 32 & $16.73 \pm 5.06$ \\
\hline
\end{tabular}

SONK: spontaneous osteonecrosis of the knee; VAS: Visual Analogue Scale; OKS: Oxford Knee Score

Table 4 Statistical comparison of pre- and postoperative outcome scores

\begin{tabular}{lllll}
\hline Preoperative - Postoperative & $95 \% \mathrm{CI}$ & $\mathrm{T}$ & p-value \\
\hline Preop VAS - Postop VAS & $(5.0700,5.7995)$ & 30.901 & $<0.001$ \\
Preop OKS - Postop OKS & $(21.2991,26.5269)$ & 18.973 & $<0.001$ \\
\hline
\end{tabular}

VAS: Visual Analogue Scale; OKS: Oxford Knee Score

\section{Figures}

\section{Patients undergo UKA in} 2013-2014 (81)

\section{Medial knee osteoarthritis without osteonecrosis lesion (34)}

Combined with other compartment osteoarthritis (3)

\section{Patients with final diagnosis of SONK (42)}

Patients with MRI-proved image data (23)
Patients without MRI image data (19) 
Figure 1

Flow diagram of the patient selection procedure including the exclusion criteria

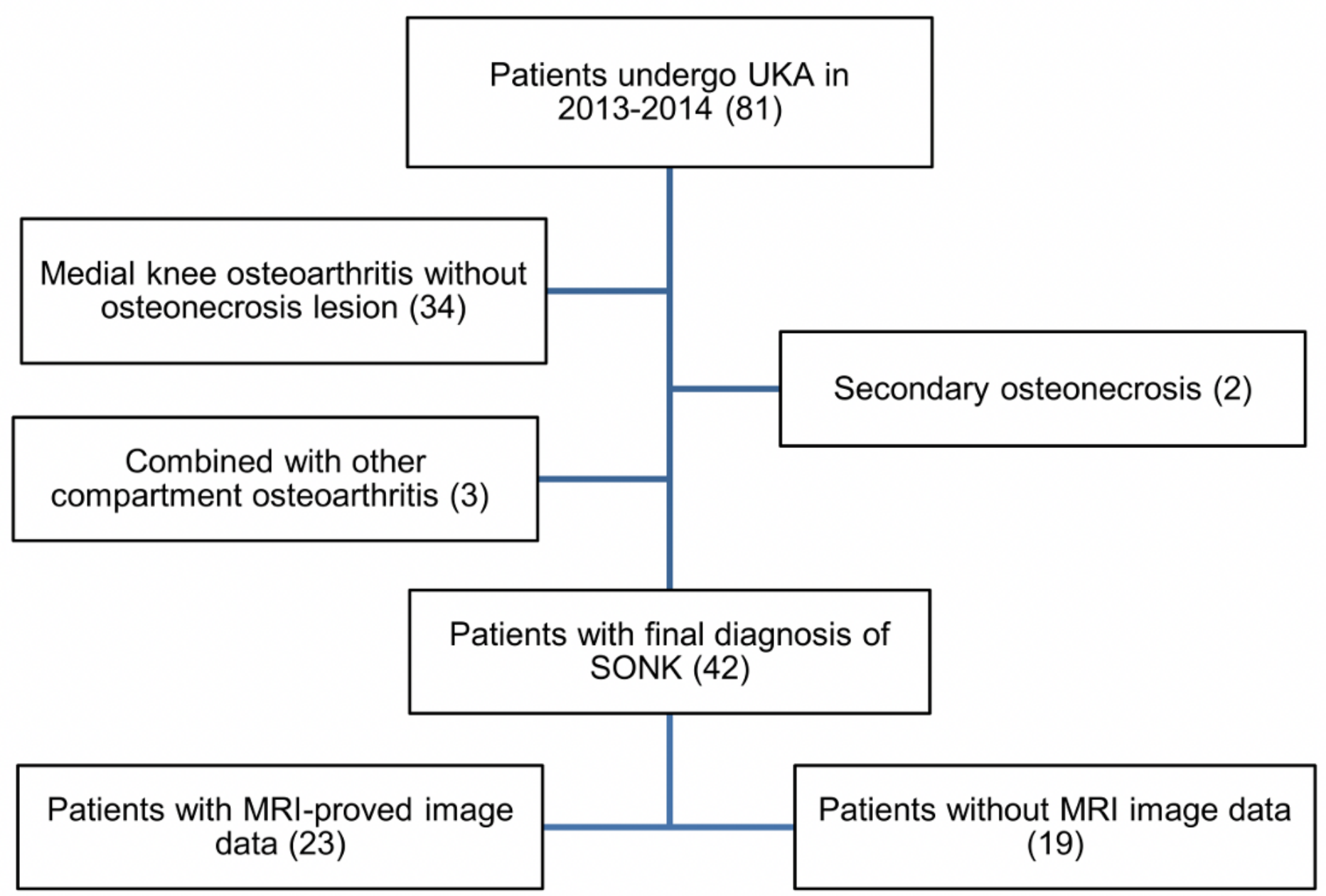

Figure 1

Flow diagram of the patient selection procedure including the exclusion criteria 

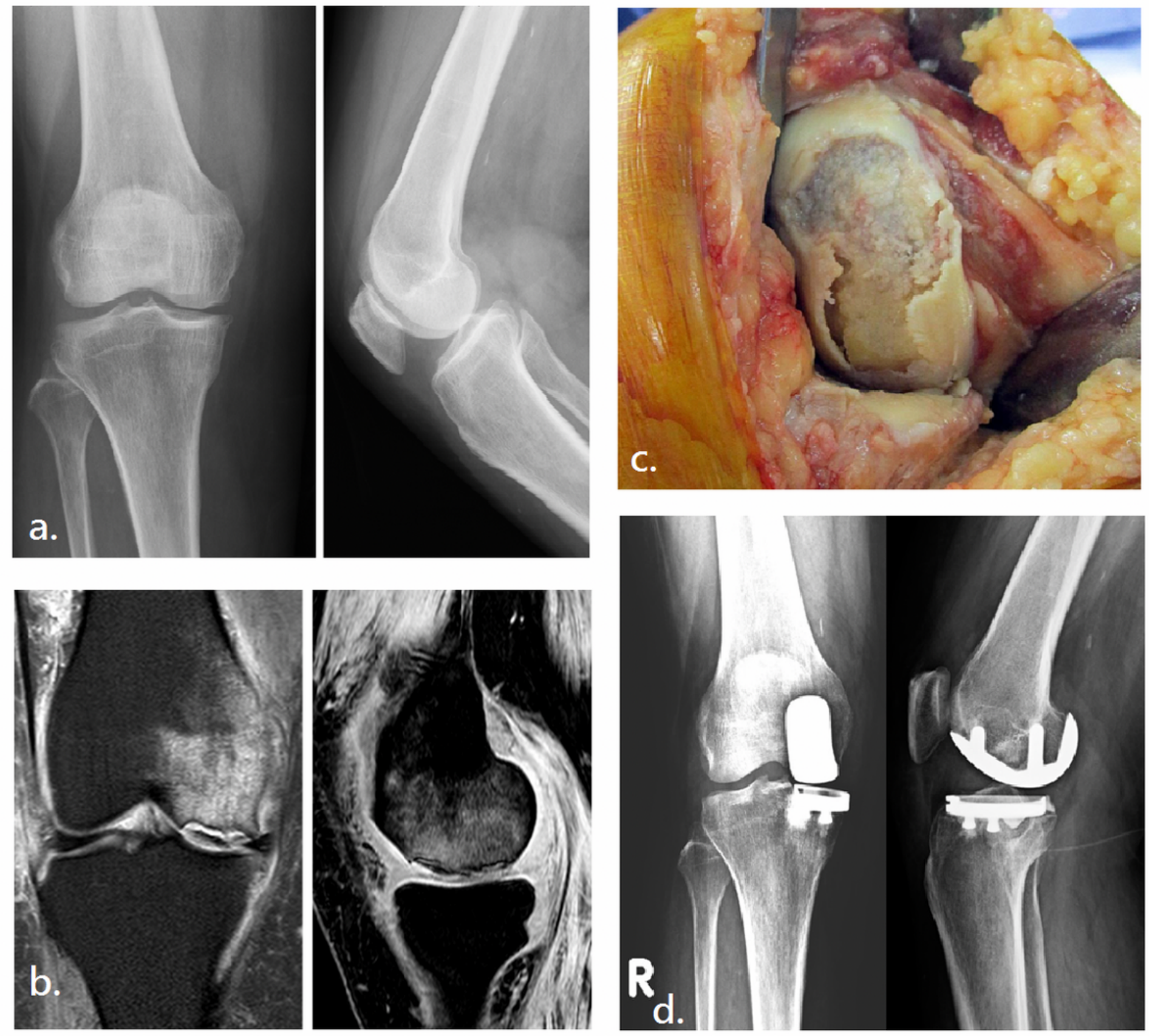

Figure 2

(a) Anteroposterior and lateral knee x-rays showing medial condyle flattening with joint space narrowing (b) T2-weighted magnetic resonance images showing osteochondral fractures of the medial femoral condyle with marked marrow edema; (c) clinical picture taken during surgery showing a massive osteochondral fracture in medial femoral condyle; (d) knee x-rays showing reconstruction status-post unicompartmental knee arthroplasty 

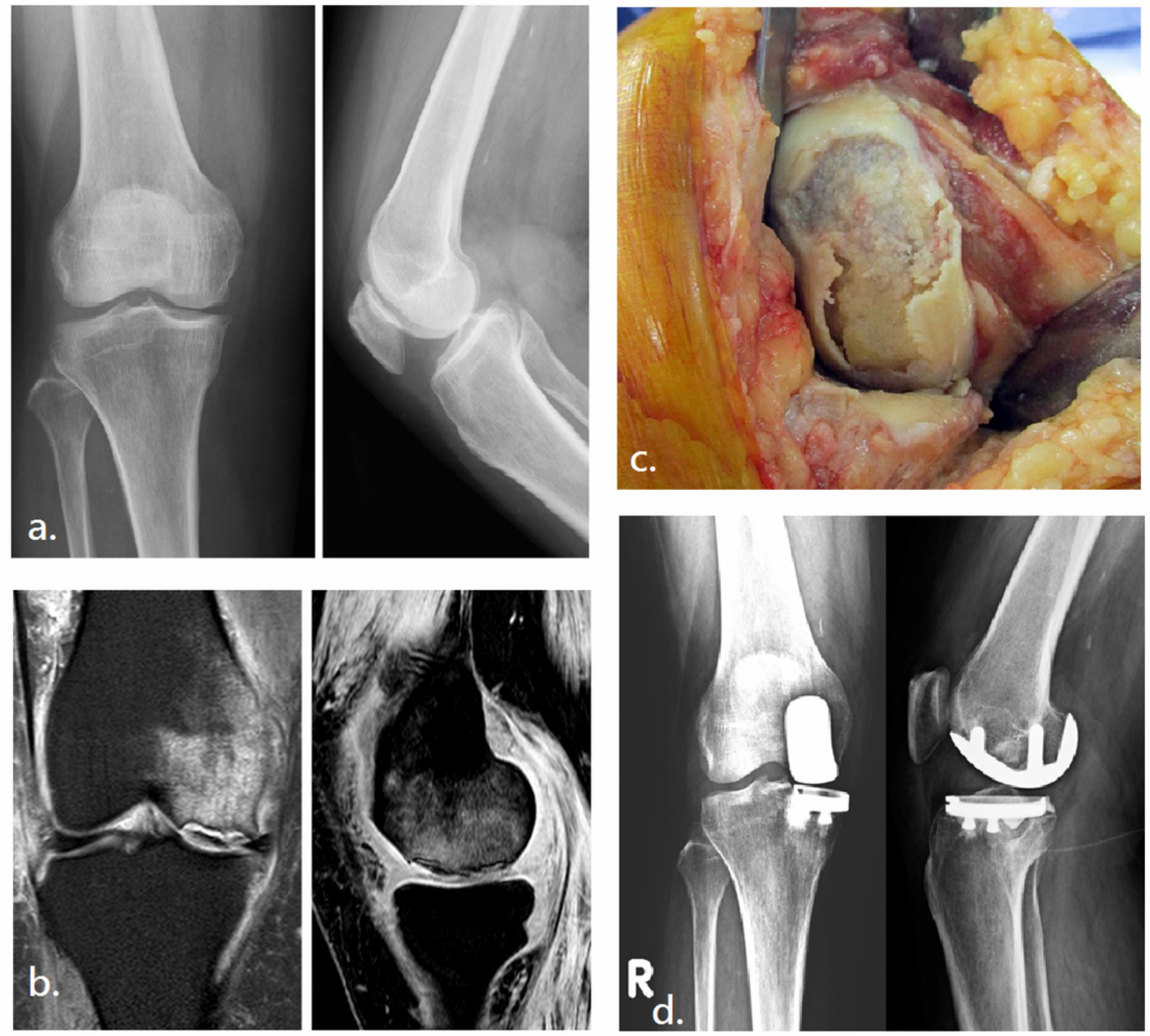

Figure 2

(a) Anteroposterior and lateral knee x-rays showing medial condyle flattening with joint space narrowing (b) T2-weighted magnetic resonance images showing osteochondral fractures of the medial femoral condyle with marked marrow edema; (c) clinical picture taken during surgery showing a massive osteochondral fracture in medial femoral condyle; (d) knee x-rays showing reconstruction status-post unicompartmental knee arthroplasty 


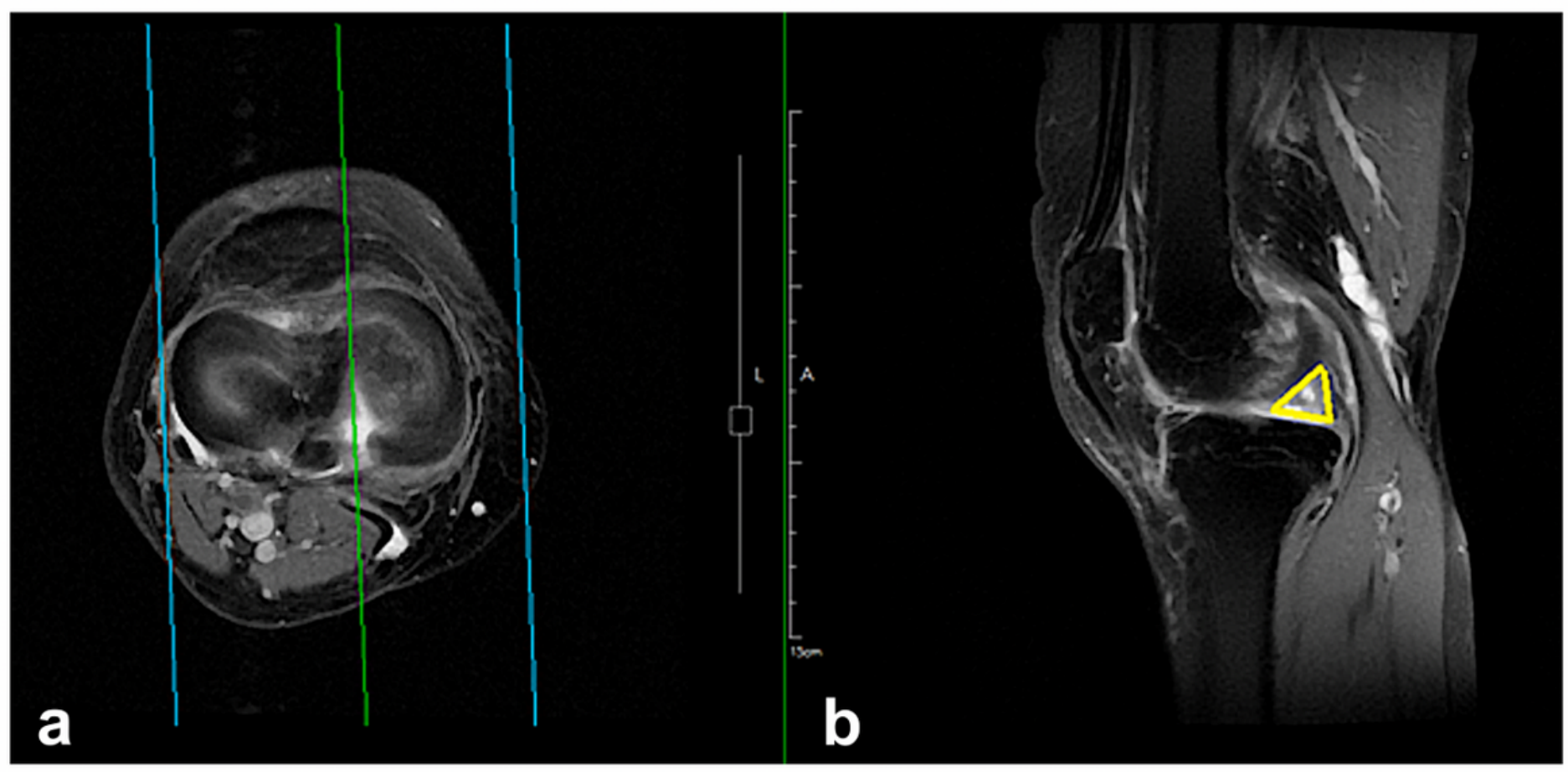

\section{Figure 3}

(a) Cleft sign: interruption of the medial meniscus at the posterior horn in axial plane; (b) ghost sign: empty image of medial meniscus
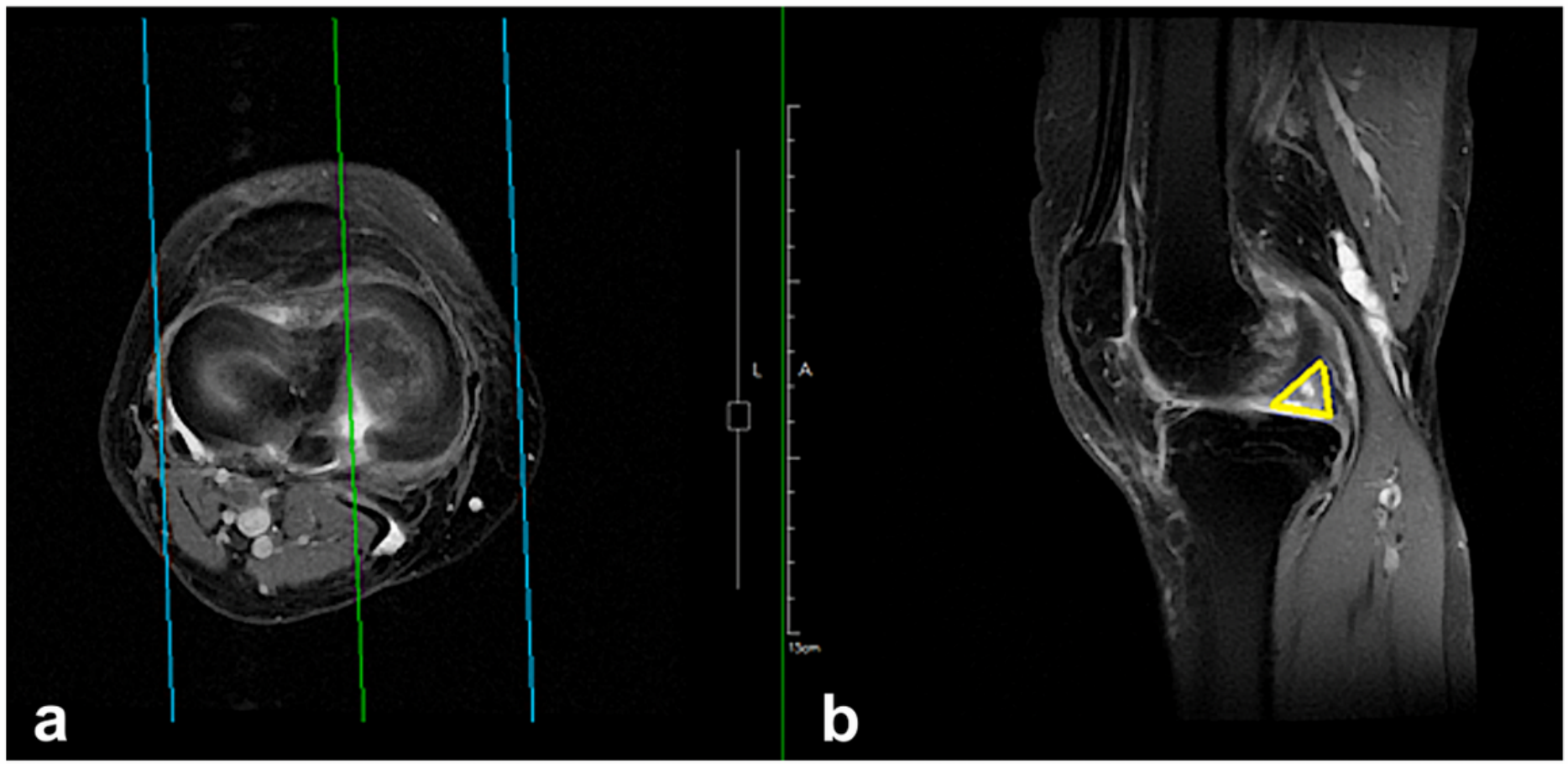

Figure 3 
(a) Cleft sign: interruption of the medial meniscus at the posterior horn in axial plane; (b) ghost sign: empty image of medial meniscus 